Seguridad y salud laboral: una revisión en el contexto actual, a propósito de la nueva ISO 45.001

\title{
Seguridad y salud laboral: una revisión en el contexto actual, a propósito de la nueva ISO 45.001
}

Occupational health and safety: a review in the current context, with regard to the new ISO 45.001

Saúde e segurança ocupacional: uma revisão no contexto atual, no que diz respeito à nova ISO 45.001

Roberto B. Oviedo-Quiñonez ${ }^{\text {I }}$

robertoviedo_q@hotmail.com

Pavel O. Defranc-Balanzategui II

paveldefranc@hotmail.com

Tomas V. Otero-Gorotiza III

totero2@hotmail.com

Recibido: 14 de septiembre de 2017 * Corregido: 05 de octubre de 2017 * Aceptado: 17 de enero de 2018

ı. Magister de Seguridad, Higiene y salud Ocupacional, Docente de la Universidad de Guayaquil, Guayaquil, Ecuador.

II. Magister de Seguridad, Higiene y salud Ocupacional, Docente de la Universidad de Guayaquil, Guayaquil, Ecuador.

III. Magister en Seguridad, Salud y Ambiente, Docente de la Universidad Central del Ecuador, Quito, Ecuador. 


\title{
Resumen
}

La gestión de la seguridad y la salud laboral se ha convertido en un tema clave tanto en el contexto empresarial como en el ambiente académico-científico. La emisión de la nueva ISO 45.001 ha dinamizado el debate, $\mathrm{y}$ ha hecho que la seguridad y la salud ocupacional sea un aspecto normativo vigente, y que alcance una posición en la agenda junto a la gestión de la calidad y la gestión ambiental. A partir de una investigación documental, en este documento se presenta una revisión del contexto actual de la seguridad y salud ocupacional. Se destaca su relación con la productividad y con el desarrollo sustentable. Así mismo, se identifican buenas prácticas en la gestión, así como sectores emergentes.

Palabras clave: seguridad; salud ocupacional; productividad; desarrollo sustentable; ISO 45.001

\begin{abstract}
The management of occupational health and safety has become a key issue both in the business context and in the academic-scientific environment. The issuance of the new ISO 45.001 has invigorated the debate, and has made safety and occupational health a current regulatory aspect, and that reaches a position on the agenda along with quality management and environmental management. Based on a documentary investigation, this document presents a review of the current context of Occupational Health and Safety. Its relationship with productivity and sustainable development stands out. Likewise, good management practices are identified, as well as emerging sectors.
\end{abstract}

Key words: safety; occupational health; productivity; sustainable development; ISO 45.001

\section{Resumo}

A gestão da saúde e segurança ocupacional tornou-se uma questão fundamental tanto no contexto empresarial quanto no meio acadêmico-científico. A emissão da nova ISO 45.001 revigorou o debate e tornou a segurança e a saúde ocupacional um aspecto regulatório atual, e que atinge uma posição na agenda juntamente com a gestão da qualidade e a gestão ambiental. Com base em uma investigação documental, este documento apresenta uma revisão do atual contexto de saúde e segurança ocupacional. Sua relação com produtividade e desenvolvimento sustentável se destaca. Da mesma forma, boas práticas de gestão são identificadas, assim como setores emergentes.

Palavras chave: segurança; saúde ocupacional; produtividade desenvolvimento sustentável; ISO 45,001 


\section{Introducción}

En el contexto actual mundial, ha habido un creciente interés en los niveles de bienestar, el cual incluye el bienestar en el trabajo (Kowalski \& Loretto, 2017). El hecho de la industrialización y el desarrollo de los sectores de prestación de servicios hoy en día se estén acelerando, ha provocado un auge del problema de salud en el lugar de trabajo, lo que ha centrado el interés en el tema de la seguridad y la salud ocupacional (SSO) como aspecto clave del bienestar humano (Jilcha \& Kitaw, 2016).

Las consecuencias de no gestionar adecuadamente los riesgos de seguridad y salud en el trabajo son evidentes. La Organización Internacional del Trabajo (OIT), estima que cada año mueren 2,3 millones de personas como resultado de accidentes y enfermedades relacionadas con el trabajo; hay alrededor de 313 millones de accidentes en el lugar de trabajo; y 160 millones de personas se ven afectadas por enfermedades ocupacionales (Jones, 2017). Por su parte, la Organización Internacional para la Normalización, mejor conocida como ISO (2018), estima que más de 2.78 millones de personas aún mueren cada año por una enfermedad o lesión relacionada con el trabajo; y agrega que, esta cifra no incluye los miles de enfermedades y lesiones industriales que cambian la vida que sufren los trabajadores diariamente.

La protección de la salud y seguridad de los trabajadores es un proceso complejo que requiere de la participación de todos los integrantes de la cadena de valor de una organización con el fin de realizar intervenciones sistémicas y multidisciplinarias que logren dar respuesta a los problemas que aquejan a los grupos laborales desde la perspectiva del trabajo prescrito y del trabajo real (Rodríguez, 2010); es así como, esta disciplina no debe verse como un área aislada, y es la alta dirección quien debe liderar este tipo de iniciativa.

Los costos económicos derivados de las condiciones de SSO a nivel empresarial, nacional y global son elevados (Gómez, 2007). Los costos humanos, sociales y económicos de los accidentes de trabajo, lesiones y enfermedades y los grandes desastres industriales han sido causa de preocupación en todos los niveles desde el lugar de trabajo individual hasta el nacional e internacional (Hori, 2012). Dichos accidentes, enfermedades y muertes en el ámbito laboral, la pérdida de la vida, el sufrimiento y la inhabilitación temporal de millones de trabajadores, suponen elevados costos en términos humanos y económicos; estos datos ponen de manifiesto una situación que es necesario abordar de manera firme y decidida desde muchas vertientes (Cobos \& Garí, 2008). 
La OIT (2005) calcula que las pérdidas debidas a indemnizaciones, días de trabajo perdidos, interrupciones de la producción, formación y reconversión profesional y gastos médicos, entre otros aspectos, representan el 4 por ciento del producto interno bruto (PIB) mundial (1.251.353 millones de dólares estadounidenses).

Las cifras mencionadas son un llamado de atención sobre el hecho de que a pesar de las diferentes políticas y medidas de seguridad social y salud que se han formulado e implementado a nivel mundial, no se ha podido garantizar el establecimiento de contextos saludables de trabajo. Sin embargo, es importante señalar que las organizaciones se preocupan constantemente por garantizar el bienestar social y la calidad de vida laboral de los trabajadores (Gómez, 2007).

Por otro lado, la SSO, como todas las facetas de los negocios, debe ser administrada adecuadamente; el sistema de SSO de una organización ayuda a garantizar un control efectivo de los riesgos inherentes y la mejora continua en su desempeño, previene enfermedades o lesiones relacionadas con el trabajo y logra el cumplimiento de las normas y los estándares (Moraru, 2012).

En este documento, se presenta una revisión del contexto actual de la seguridad y salud ocupacional, laboral o del trabajo, a propósito de la emisión de la nueva norma ISO 45.001 (2018). El enfoque de la investigación es documental, basada en la revisión de literatura de los últimos años. Para este fin, se revisaron recursos como Web of Science ${ }^{\circledR}$, Scopus ${ }^{\circledR}$, Google Scholar ${ }^{\circledR}$, entre otros, y como descriptores de búsqueda, se emplearon salud, seguridad, e ISO 45.001, entre otros.

Las buenas prácticas en la gestión de la seguridad y la salud ocupacional conducen a mejores niveles de satisfacción laboral entre los empleados de una organización (Sembe \& Ayuo, 2017). El problema global para el desarrollo sostenible del ciudadano es la salud y los grupos de trabajo seguros en su lugar de trabajo; para lograr esto, uno de ellos es el desarrollo de entornos sostenibles de SSO (Jilcha \& Kitaw, 2017).

La publicación de ISO 45001, un conjunto único de requisitos internacionales para ayudar a las organizaciones a proteger a los trabajadores de daños, está diseñada para combatir este problema (ISO, 2018). La introducción de la norma ISO 45001 también brinda la oportunidad de establecer relaciones de trabajo más estrechas entre los profesionales de SSO y la gerencia, y para que las organizaciones demuestren un liderazgo más visible y socialmente responsable (Jones, 2017).

En este documento, se presenta un preámbulo conceptual para a fundamentar los argumentos siguientes; se analiza la relación de la seguridad y salud ocupacional con la productividad y con el 
desarrollo sustentable; así mismo, identifican las buenas prácticas y los sectores emergentes, para concluir con reflexiones finales.

\section{Un preámbulo conceptual}

El estudio del bienestar en el trabajo es un área multidisciplinaria de interés de investigación, que abarca una serie de disciplinas, incluida la economía, la psicología industrial y organizacional, la antropología y la sociología; como tal, conceptualizar exactamente a qué se refiere el bienestar puede ser complejo (Kowalski \& Loretto, 2017).

La historia de la salud y seguridad en el trabajo ha estado marcada por determinantes sociales, políticos y económicos propios de cada época, trayendo consigo diferentes formas de concebir, realizar el trabajo y proteger la salud de los trabajadores (Carvajal \& Molano, 2012). La seguridad y la salud en el trabajo son cuestiones controvertidas importantes para tener éxito en el desarrollo industrial (Jilcha \& Kitaw, 2017). La salud laboral y los accidentes son producto de múltiples causas: persona, puesto de trabajo, factores organizacionales y aspectos inter-organizacionales (Díaz et al, 2008).

La seguridad y salud en el trabajo, se ha planteado como un enfoque integrador a los diferentes corrientes, siendo más incluyente, facilitando un entramado de actividades que persiguen un mismo fin: el bienestar y calidad de vida de los trabajadores, quienes se convierten en agentes fundamentales para la transformación de las condiciones de trabajo (Carvajal \& Molano, 2012).

De acuerdo con Rodríguez (2010), la protección de la salud y seguridad de los trabajadores se logra a través de tres grandes áreas de conocimiento que, de manera entrelazada, permiten adecuar el medioambiente y los métodos de trabajo a las capacidades de los individuos: ergonomía, higiene y seguridad industrial.

La cultura de la SSO, es un concepto complejo que ha sido objeto de una amplia investigación desde los años ochenta, y existen diferentes perspectivas sobre cómo definir y abordar esta cultura en las organizaciones (European Agency for Safety and Health at Work (EU-OSHA), 2011)

La SSO, o OSH por sus siglas en inglés, se define como la ciencia de la anticipación, el reconocimiento, la evaluación y el control de los peligros que se originan en el lugar de trabajo y que podrían perjudicar la salud y el bienestar de los trabajadores, considerando su posible impacto en el entorno general (Alli, 2008). Es una disciplina que trata de la prevención de las lesiones y enfermedades relacionadas con el trabajo, y de la protección y promoción de la salud de los trabajadores (OIT, 2011). 
La OIT (2011), la presenta como un conjunto de herramientas lógicas, caracterizadas por su flexibilidad, que pueden adaptarse al tamaño y a la actividad de la organización y centrarse en los peligros y riesgos generales o específicos asociados con dicha actividad.

Para los empleadores, gerentes comerciales y profesionales de SSO que luchan por la excelencia en el campo de la seguridad y salud laboral, la cuestión clave es garantizar que se eviten tanto los accidentes laborales y las enfermedades relacionadas con el trabajo como sea posible, y que todos los comportamientos seguros y saludables empleados es promovido. (European Agency for Safety and Health at Work (EU-OSHA), 2011).

La moderna concepción de la SSO, se diferencia de sus precedentes en la enorme amplitud de sus objetivos y en la gran ambición de sus instrumentos; este nuevo enfoque trasciende la simple evitación o reducción de los riesgos para abarcar una política global de mejora de las condiciones de trabajo en que se desenvuelve el trabajador, basada en la visión unitaria de la organización, donde quede integrada: la planificación, la coordinación, la participación, el control de la eficacia, la información y la formación (Romeral, 2012).

Una cuestión clave que rodea a la investigación en este campo, es que sigue habiendo una falta de consenso sobre lo que es el bienestar y la fusión con otros términos, como el compromiso de los empleados (Kowalski \& Loretto, 2017).

Muchos autores reconocen la evidencia de una relación entre las prácticas de recursos humanos y el bienestar de los empleados, sin embargo, la mayoría observa que la relación no está clara (Kowalski \& Loretto, 2017).

\section{La seguridad y salud ocupacional y la productividad de las organizaciones}

En el escenario socioeconómico actual, se concibe a la empresa con criterios globales que incluyen los de calidad, prevención de riesgos laborales y medio ambiente (Romeral, 2012). En toda organización es preciso respetar principalmente la condición humana, dado que ninguna podría ser tal sin la presencia del hombre; sólo teniendo en cuenta primero al ser humano será posible atender todos los aspectos inherentes a la producción y a la productividad (Nicolaci, 2008).

Así, la comprensión del recurso humano como el más importante de todos los activos, en la gestión contemporánea, puede basarse en su papel inevitable en el manejo de todos los demás activos o recursos de la organización para la productividad (Akpan, 2011). Por lo que, una de las implicaciones organizacionales del pobre bienestar de los empleados es el potencial efecto adverso sobre la 
productividad (Kowalski \& Loretto, 2017). En síntesis, hombre, organización, condiciones y medio ambiente de trabajo, producción y productividad están estrechamente relacionados entre sí y conforman un todo en el cual cada uno es un elemento esencial en la interacción con los demás (Nicolaci, 2008).

El decrecimiento de recursos podría originar en el puesto de trabajo un mayor número de accidentes, enfermedades, víctimas mortales y mala salud a causa del desempleo, con consecuencias serias en los gastos de la seguridad social y en la productividad (Moreno \& Godoy, 2012). La accidentalidad laboral tiene consecuencias sobre la productividad y competitividad de las empresas y sobre la sociedad en su conjunto (Riaño, Hoyos \& Valero, 2016).

Por eso, actualmente es más significativo y relevante apoyar los esfuerzos que puedan realizar las organizaciones para fomentar la seguridad y los riesgos laborales, a objeto de garantizar la implementación adecuada de estándares de seguridad y salud ocupacional (Moreno \& Godoy, 2012).

\section{Seguridad, Salud Ocupacional y Desarrollo Sustentable}

Las economías modernas están impulsadas por la agricultura, la industria manufacturera y las industrias de servicios; hoy en día, la salud y la seguridad se están convirtiendo en los temas clave de las preocupaciones globales (Jilcha \& Kitaw, 2017). Los objetivos de estas disciplinas son la protección y promoción de la salud de los trabajadores y la contribución a un desarrollo seguro y sostenible (Rodríguez, 2010).

El desarrollo sustentable y la seguridad, la salud y el medio ambiente en el trabajo son dos conceptos controvertidos que han atraído la atención de muchos investigadores en los últimos años (Zohreh \& Napsiah, 2014). El cambio dinámico en la innovación en materia de salud y seguridad en el lugar de trabajo, la alta prevalencia de enfermedades profesionales y el registro de accidentes son los factores iniciales del desarrollo sostenible. La existencia de problemas de seguridad y salud en el trabajo está agregando un nivel de pobreza a la carga existente de la sociedad (Jilcha \& Kitaw, 2017).

El desarrollo sustentable, por un lado, tiene un punto de vista holístico general para mantener el mundo como un lugar habitable para la próxima generación, al tiempo que se logra una calidad de vida satisfactoria para las comunidades actuales, mientras que la seguridad, la salud y el medio ambiente en el trabajo se centran principalmente en el bienestar de la fuerza de trabajo, pero sus efectos y consecuencias pueden abarcar a toda la sociedad y al mundo entero, ambos tiene como deseo genuino es el bienestar y la satisfacción de las personas (Zohreh \& Napsiah, 2014). 
La esencia de la seguridad, la salud y el medio ambiente ocupacionales y el desarrollo sostenible representan la existencia de una relación mutua entre estos dos conceptos críticos; tienen los mismos objetivos y se ocupan de los mismos problemas, que es el bienestar y el bienestar constantes de los seres humanos, que se examinan desde diferentes ángulos y se definen en terminologías diferentes; además, la ambición final de estas dos políticas es mantener la salud mental y física continua de las personas (Zohreh, \& Napsiah, 2014).

Como lo señalan Amponsah-Tawiah \& Dartey-Baah (2011), hasta la fecha, la salud ocupacional no ha alcanzado un alto perfil en la agenda de desarrollo sostenible, especialmente en los países en desarrollo, donde la mayoría de la gente se dedica a las industrias primarias, como la agricultura, la explotación maderera y la minería. hasta ahora ningún país ha tenido éxito en alcanzar este objetivo para todos los trabajadores.

Dado que la seguridad, la salud y el medio ambiente en el trabajo y el desarrollo sostenible se refieren a los mismos problemas, la integración de los resultados de sus investigaciones y la adopción de un enfoque común para ambos conceptos serían de gran importancia (Zohreh, \& Napsiah, 2014).

\section{Nuevos factores que impactan la seguridad y salud laboral}

La naturaleza del trabajo y la forma en que se conceptualiza ha ido evolucionando desde los albores de la humanidad; a medida que las sociedades han pasado de la reunión de cazadores a una base agraria y luego a la vida urbana, las actividades que las personas han emprendido han cambiado (Litchfield, Cooper, Hancock \& Watt, 2016).

Los peligros para la seguridad y la salud en el lugar de trabajo hoy en día se consideran una fuerza motriz para encontrar soluciones para prevenir las consecuencias negativas para los empleados de las industrias manufactureras (Jilcha \& Kitaw, 2016).

Sparks, Faragher \& Cooper (2001), discuten las condiciones de los contextos de trabajo y su incidencia en el bienestar y la salud de los trabajadores alrededor de aspectos como: la inseguridad en el trabajo, los turnos laborales, el control sobre el trabajo y el estilo de dirección; sin embargo, este planteamiento ha cambiado en las últimas dos décadas, a la luz de nuevos factores que vienen afectando las condiciones en las cueles se desempeñan los trabajadores.

El ritmo del cambio ha aumentado exponencialmente y la revolución de la tecnología de la información ha transformado el trabajo para muchos en menos de una generación (Litchfield, Cooper, Hancock \& Watt, 2016). El crecimiento en el uso de la tecnología de la información en el trabajo, la 
globalización de muchas industrias, la reestructuración organizativa, los cambios en los contratos de trabajo y la programación del horario de trabajo han transformado radicalmente la naturaleza del trabajo en muchas organizaciones (Sparks, Faragher \& Cooper, 2001).

La introducción de una nueva tecnología afecta la salud de los trabajadores; por ejemplo, la introducción de ordenadores o pantallas de visualización de datos en un número cada vez mayor, ha generado preocupación por sus efectos negativos sobre la salud de los trabajadores. Además, los procesos de automatización del trabajo pueden incrementar la exigencia psíquica de manera importante, al disminuir la autonomía del trabajador y aumentar la demanda de la tarea (Gómez, 2007).

La fuerza de trabajo en sí también se está diversificando, con un aumento en la participación femenina, un número creciente de parejas de doble ingreso y trabajadores de mayor edad (Sparks, Faragher \& Cooper, 2001).

Entre las nuevas realidades del trabajo se pueden señalar también los fenómenos de la desaparición y las fusiones de las empresas, lo cual da lugar a la pérdida de estabilidad, la incertidumbre, la pérdida de control y a la necesidad de replantear los proyectos de vida personales y familiares (Gómez, 2007). Retos de la gestión de la seguridad y salud laboral en espacios y economías emergentes

La SSO es un área interdisciplinaria que se centra en la seguridad y la salud de los trabajadores; el ambiente de trabajo y las condiciones generales en la sociedad actual están en constante cambio, a pesar de que la importancia de este campo particular de seguridad y salud ocupacional ha permanecido igual; sin embargo, se han creado una amplia gama de nuevos problemas debido al rápido desarrollo de la ciencia y la tecnología en las industrias (Hori, 2012).

En las economías emergentes, la seguridad y la salud en el trabajo se han pasado por alto en sus políticas y estrategias de desarrollo industrial; en su mayoría se centran en el volumen de producción o beneficio que socava el efecto latente de un entorno de trabajo insatisfactorio (Jilcha \& Kitaw, 2016). La mayor parte del enfoque de la compañía se centra en la satisfacción del cliente externo con su producto o servicio sin tener en cuenta la satisfacción de los trabajadores y la comodidad del entorno de trabajo en los países con rezago económico (Jilcha \& Kitaw, 2016).

La gestión de la SSO los trabajadores del sector de la construcción, se ha convertido en un tema muy importante para las partes interesadas, que incluyen las organizaciones, los gobiernos y la sociedad en general, dado el impacto significativo en la salud y la seguridad de los trabajadores (Suárez, Carvajal \& Catalá, 2017). Este sector de la industria juega un papel importante en la contribución a la economía 
y el desarrollo a nivel mundial (Jaafar, et al., 2017); en la mayoría de los países industrializados, esta importancia se refleja en la contribución al producto interno bruto (PIB) (Suárez, et al. 2017). Dada la naturaleza compleja del proceso de construcción, accidentes son muy frecuentes en este sector, lo que deriva en altos índices de mortalidad y niveles de riesgo elevados, convirtiéndolo en una industria insegura.

Además de las contribuciones de Suárez, et al. (2017), y Jaafar, et al. (2017), destaca también el trabajo de Cepeda, Munguía \& Velásquez (2015), quienes, a partir de un estudio de caso, evalúan la importancia de la seguridad y la salud en el trabajo en los sistemas de gestión en esta industria de la construcción.

Por otra parte, las nuevas tecnologías, como los robots colaboradores, pueden ser la fuente de nuevos accidentes, y podrían estar en el origen de una exposición hasta ahora desconocida a sustancias peligrosas, como los nanomateriales; los ataques de piratas informáticos y la manipulación de datos se vuelven más peligrosos en los sistemas de producción y en lugares de trabajo con un alto grado de interconexión (Reinert, 2016).

Otro fenómeno actual es el problema del envejecimiento de la fuerza de trabajo, la cual ha venido aumentando; las personas mayores incluyen una variedad de funciones fisiológicas debido a la edad en general, sin embargo, con el estrés adicional creado por entornos de trabajo insalubres, hace que este problema sea tomado en cuenta en la agenda (Hori, 2012).

Gómez \& Turizo (2016), quienes sustentándose en datos de la Organización Mundial de la Salud (OMS), destacan la relevancia de abordar desde la esfera jurídica y social la discapacidad y analizan la evolución normativa en riesgos laborales, la relación del concepto de trabajo en relación con las personas con discapacidad, la implementación del Sistema de Gestión de la seguridad y salud laboral, y plantean estrategia de ajustes razonables como un asunto de la seguridad y salud para los trabajadores con discapacidad.

Ahmed, et al. (2017), quienes cubren el estado actual de las prácticas relacionadas con la SSO en el segmento de la construcción informal de Pakistán; encontrando que tanto los empleadores como los trabajadores carecen de conocimiento del marco legal y normativo de SSO y, además, en ese contexto, no se promulgan prácticas de esta naturaleza. Agregan que, los accidentes relacionados con el trabajo, no reciben la debida atención y no existe un sistema formal de reportes de los mismos; que esta industria, emplea a una gran parte de la fuerza laboral informal, y que la falta de SSO incurre en un alto costo humano. Los autores señalan que, los hallazgos de la investigación pueden desempeñar su 
Seguridad y salud laboral: una revisión en el contexto actual, a propósito de la nueva ISO 45.001

papel en el fortalecimiento de los argumentos a favor de las reformas en el sector, y que, podrían permitir a los empleadores del sector aumentar su conocimiento sobre las prácticas de SSO y, como resultado, tratar de ofrecer entornos más seguros para sus trabajadores.

Garnica \& Barriga (2018), quienes determinan las principales barreras para la implementación de sistemas de gestión de salud y seguridad ocupacional OHSMS en el contexto de pequeñas empresas brasileñas desde la perspectiva de propietarios / gerentes, auditores laborales y consultores de OHS, encontrando que los propietarios / gerentes tienden a culpar a los empleados y al gobierno por la dificultad de implementar OHSMS, y los actores externos tienden a culpar a la administración y la asignación de recursos; y que, las opiniones convergen en el comportamiento inadecuado de la gestión, la información ineficaz y la comunicación y la priorización de la producción. Estas barreras deberían superarse no solo para facilitar la implementación de OHSMS sino también para mejorar las condiciones de gestión de todas las operaciones de pequeñas empresas.

Entre otras investigaciones que abordan espacios emergentes, se destacan autores como Ove-Hansson \& Schenk (2016), quienes tratan el problema de la regulación del embarazo y la salud ocupacional, y la discriminación de las mujeres; Sembe \& Ayuo (2017), quienes evalúan el efecto de algunas prácticas de gestión de la seguridad y la salud en el trabajo sobre la satisfacción laboral de los empleados en los campus universitarios; Rojas, et al. (2014), quienes plantean un modelo de gerencia estratégica para un servicio de seguridad y salud en el trabajo de Tipo Mancomunado; Riaño, Hoyos \& Valero (2016), quienes analizan la evolución de los SSSO y sus impacto en la accidentabilidad laboral en el sector petroquímico; Elgstrand \& Vingård (2013), quienes presentan una antología de la situación de Seguridad y Salud Ocupacional en Minería; entre otros.

En el contexto ecuatoriano, se destacan los trabajos de Cajías, Álvarez, Merino \& Gómez, (2017), quienes a partir de los resultados del análisis del estado actual de SST en Ecuador, concluyen éste es preocupante y enfrenta un problema peligroso que continuará en las próximas décadas. Agregan que, es necesario abordar la persistencia de un alto número de trabajadores informales, el envejecimiento de la población activa, el aumento de los accidentes del trabajo y la falta de conocimientos sobre enfermedades profesionales; y que, además, las autoridades de control deben revisar la legislación obsoleta existente debido a cambios sociales, demográficos y económicos en curso, y deben liderar, promover y fomentar una cultura de seguridad de la salud en el trabajo a nivel nacional y evitar la reducción de los programas académicos acreditados en este campo, lo que resulta en una investigación 
Seguridad y salud laboral: una revisión en el contexto actual, a propósito de la nueva ISO 45.001

científica insuficiente en SST. Todos estos hallazgos se oponen al Programa de Trabajo Decente de la OIT, previsto para 2030, que evidencia la necesidad de diseñar un Plan Nacional de SST.

\section{Acerca de la nueva ISO 45.001}

Los antecedentes de la ISO 45.001 se pueden ubicar en el Instituto Británico de Normalización (British Standards Institute, BSI), que propuso a ISO en 2013 que OHSAS 18001 se considerara como la base para un estándar internacional; ISO acordó y estableció un comité para supervisar su desarrollo (Jones, 2017).

Sobre la base del éxito de OHSAS 18001, dirigido por el Reino Unido, y de muchos documentos nacionales excelentes, ISO 45001 es el primer estándar del sistema de gestión de salud y seguridad ocupacional. Combina el rigor técnico de los estándares $\mathrm{OH} \& \mathrm{~S}$ ya utilizados en todo el mundo con el marco ISO común de estándares populares como ISO 9001 e ISO 14001, lo que simplifica la integración de los usuarios en su gestión comercial general. ISO 45001 ha sido desarrollado por expertos de 69 países en colaboración con representantes de organizaciones clave como la Organización Internacional del Trabajo (OIT), la Confederación Sindical Internacional (CSI) y la Organización Internacional de Empleadores (OIE) (ISO, 2018).

Aunque de reciente publicación, la norma ISO 45.001 ya ha tenido importantes referentes para su discusión a partir de los borradores disponibles en años anteriores; entre estos referentes se puede mencionar a Chiquito, Loor \& Rodríguez (2016), quienes realizan una revisión bibliografía para determinar la relación que puede estar relacionados entre las normas OHSAS 18001:2007 y las ISO 45001 como parte del proceso de transición, Permitiendo a las organizaciones que ya tiene implementado el sistema de gestión de seguridad y salud en el trabajo puedan tener un conocimiento de las semejanzas y diferencias de estas normas; Jones (2017), quien destaca las implicaciones y la importancia de la nueva norma ISO 45001 para abordar las fallas mundiales de salud y seguridad, y aborda la historia de los sistemas de gestión de seguridad y salud ocupacional (OHSMS); el papel de los estándares internacionales; el proceso de normas ISO; progreso en ISO 45001; y los objetivos para la gestión de la SST en todo el mundo.

Las diferencias entre OHSAS 18001 e ISO 45001 podrían significar que existen implicaciones para las organizaciones que buscan la certificación y sus asesores; y también para los auditores que están auditando según el nuevo estándar. Estos incluyen el requisito de la demostración de liderazgo y la consideración adecuada del contexto de una organización. (Jones, 2017). 
Seguridad y salud laboral: una revisión en el contexto actual, a propósito de la nueva ISO 45.001

Las Directrices son un método lógico, propuesto como la mayoría de sistemas de gestión, a partir de la teoría de la calidad total y la aplicación del principio de la mejora continua, proceso basado en el Ciclo de Shewhart "Planificar - Hacer - Verificar - Ajustar" conocido como ciclo PHVA de Deming.

\section{Reflexiones finales}

Como lo señalan Moreno \& Godoy (2012), los desafíos a nivel global determinan un nuevo contexto para la práctica de la salud y seguridad en el trabajo, en consecuencia, se deberán desarrollar programas estratégicos innovadores y sustentables en las organizaciones, que les permitan hacerlas más eficientes, competitivas y productivas.

Teniendo en cuenta estos desafíos, es obligatorio responder a las preguntas sobre cómo la seguridad en el trabajo y la innovación de la salud da como resultado el desarrollo sostenible de los ciudadanos y avanzar en las vías hacia el desarrollo sostenible en la seguridad y salud laboral (Jilcha \& Kitaw, 2017). La gerencia requiere enfrentarlos con estrategias preventivas de cara a los nuevos y emergentes riesgos, relacionados con cambios tecnológicos, turnos en los modelos de empleo, condiciones de trabajo y la mayor vulnerabilidad de la mano de obra ante situaciones ambientales adversas en algunos sitios de labores (Moreno \& Godoy, 2012).

El cambio dinámico en la innovación en materia de salud y seguridad en el lugar de trabajo, la alta prevalencia de enfermedades profesionales y el registro de accidentes son los factores iniciales del desarrollo sostenible; la existencia de problemas de seguridad y salud en el trabajo está agregando un nivel de pobreza a la carga existente de la sociedad (Jilcha \& Kitaw, 2017).

Dado el carácter global, la velocidad y la complejidad de los cambios emergentes en la SSO, sería aconsejable que la comunidad internacional de esta área, una fuerza, siempre que sea posible, para seguir el ritmo del desarrollo, hacer uso de sus activos en beneficio de la seguridad y la salud en el trabajo y compartir ideas para la prevención de cualquier riesgo nuevo o emergente (Reinert, 2016). Aunque con base en un contexto particular, pudiera generalizarse el planteamiento de Cobos \& Garí (2008), en cuanto a que en la actualidad se está realizando un importante esfuerzo en materia de formación, que, sin embargo, su impacto real en la mejora de las condiciones de trabajo no está siendo suficientemente evaluado. Sostienen estos autores que, existe un consenso generalizado entre los diferentes agentes implicados en considerar la formación como uno de los elementos fundamentales a la hora de hacer salud laboral en las empresas., y que, la formación que actualmente se imparte no responde, en muchos casos, a las necesidades reales de los trabajadores y, en general, de la 
organización, por lo que, se hace necesario replantear los contenidos, enfoques y metodologías de la formación. la formación en materia preventiva tiene que ser para todos.

Por lo tanto, la infraestructura y los programas de salud ocupacional deberían desarrollarse más en todos los países, empleando un enfoque no legislativo para complementar los requisitos legales existentes (Amponsah-Tawiah \& Dartey-Baah (2011).

Importante que la nueva ISO 45001, reconoce la complejidad de la salud y seguridad ocupacional en el siglo XXI, donde las tecnologías e industrias emergentes son reveladoras nuevos desafíos (ISO, 2018).

Así mismo, debe considerarse en el futuro más investigación sobre efectos positivos de los nuevos factores que afectan la SSO, así como los problemas psicológicos y físicos en el lugar de trabajo.

\section{Referencias Bibliográficas}

Ahmed, I.; Shaukat, M.; Usman, A.; Nawaz, M. \& Nazir, M. (2018). Occupational health and safety issues in the informal economic segment of Pakistan: a survey of construction sites. International Journal of Occupational Safety and Ergonomics, 24(2), 240-250. DOI: https://doi.org/10.1080/10803548.2017.1366145

Akpan, E. (2011). Effective safety and health management policy for improved performance of organizations in Africa. International Journal of Business and Management, 6(3), 159-165. DOI: http://dx.doi.org/10.5539/ijbm.v6n3p159

Alli, B. (2008). Fundamental principles of occupational health and safety. Geneva: ILO- International Labour Office.

Amponsah-Tawiah, K. \& Dartey-Baah, K. (2011). Occupational Health and Safety: Key Issues and Concerns in Ghana. International Journal of Business and Social Science, 2(14), 119-126. Recuperado de http://www.ijbssnet.com/journals/Vol._2_No._14\%3B_July_2011/14.pdf

Cajías, P.; Álvarez, D.; Merino, P. \& Gómez, A. (2017). Seguridad y salud laboral en Ecuador Occupational Safety and Health in Ecuador. INNOVA Research Journal, 2(12), 139-152. Recuperado de http://www.journaluidegye.com/magazine/index.php/innova/article/view/322 Carvajal, D. \& Molano, J. (2012). Aporte de los sistemas de gestión en prevención de riesgos laborales a la gestión de la salud y seguridad en el trabajo. Movimiento científico, 6(1), 158-174. Recuperado de http://revistas.iberoamericana.edu.co/index.php/Rmcientifico/article/view/167/139 
Seguridad y salud laboral: una revisión en el contexto actual, a propósito de la nueva ISO 45.001

Chiquito, Loor \& Rodríguez (2016). Sistema de Seguridad y Salud en el Trabajo. Transición de las OHSAS 18001:2007 a la nueva ISO 45001. Revista Publicando, 3(9), 638-648. Recuperado de https://www.rmlconsultores.com/revista/index.php/crv/article/view/389/pdf_243

Cobos, D. \& Garí, A. (2008). Formación de los trabajadores para la prevención de riesgos laborales en la empresa: percepciones de los agentes sociales de la comunidad de Madrid. Escuela Abierta, 2 , 87-107. Recuperado de http://www.ceuandalucia.es/escuelaabierta/pdf/articulos_ea $11 \% 20$ pdf/formacion $\% 20$ trabajadore $\% 2$ 087 107.pdf

Díaz-Cabrera, D.; Isla-Díaz, R.; Rolo-González, G.; Villegas-Velásquez, O.; Ramos-Sapena, Y.; Hernández-Fernaud, E. (2008). La salud y la seguridad organizacional desde una perspectiva integradora. Papeles del Psicólogo, 29(1), 83-91. Recuperado de http://www.redalyc.org/pdf/778/77829110.pdf

Elgstrand, K. \& Vingård, E. (2013). Occupational Safety and Health in Mining. Anthology on the situation in 16 mining countries. Suecia: University of Gothenburg. Recuperado de https://gupea.ub.gu.se/bitstream/2077/32882/1/gupea_2077_32882_1.pdf

European Agency for Safety and Health at Work -EU-OSHA- (2011). Occupational Safety and Health culture assessment - A review of main approaches and selected tools. Luxembourg: Publications Office of the European Union.

Garnica, G. \& Barriga, G. (2018). Barriers to occupational health and safety management in small Brazilian enterprises. Production, 28, 1-10. DOI: http://dx.doi.org/10.1590/0103-6513.20170046 Gómez, I. (2007). Salud laboral: una revisión a la luz de las nuevas condiciones del trabajo.

Universitas $\quad$ Psychologica, 6(1), 105-113. Recuperado de http://www.redalyc.org/articulo.oa?id=64760111

Gómez, N. \& Turizo, F. (2016). Seguridad y salud en el trabajo en Colombia: retos frente a las personas con discapacidad. Revista CES Derecho, 7(2), 84-94. Recuperado de http://www.scielo.org.co/pdf/cesd/v7n2/v7n2a07.pdf

Hori, M. (2012). The Trend and Issues of Occupational Safety and Health in Japan. Procedia Engineering, 43, 610-614. DOI: http://dx.doi.org/10.1016/j.proeng.2012.08.107

ISO (2018). Are you ready for ISO 45001? ISOfocus, 
Seguridad y salud laboral: una revisión en el contexto actual, a propósito de la nueva ISO 45.001

Jaafar, M.; Arifin, K.; Aiyub, K.; Razman, M.; Ishak, M. \& Samsurijan, M. (2017). Occupational safety and health management in the construction industry: a review. International Journal of Occupational Safety and Ergonomics, DOI: https://doi.org/10.1080/10803548.2017.1366129

Jilcha, K. \& Kitaw, D. (2016). A literature review on global occupational safety and health practice \& accidents severity. International Journal for Quality Research, 10(2), 279-310. DOI: https://doi.org/10.18421/IJQR10.02-04

Jilcha, K. \& Kitaw, D. (2017). Industrial occupational safety and health innovation for sustainable development. Engineering Science and Technology, an International Journal, 20(1), 372-380. DOI: https://doi.org/10.1016/j.jestch.2016.10.011

Jones, K. (2017). ISO 45001 and the evolution of occupational health and safety management systems. IOSH- Institution of Occupational Safety and Health Paper, 1-9. Leicestershire, UK. Recuperado de https://www.asse.org/assets/1/7/IOSH_Article_for_ISO_45001.pdf

Kowalski, T. \& Loretto, W. (2017). Well-being and HRM in the changing workplace. The International Journal of Human Resource Management, 28(16), 2229-2255, DOI: https://doi.org/10.1080/09585192.2017.1345205

Litchfield, P.; Cooper, C.; Hancock, C. \& Watt, P. (2016). Work and Wellbeing in the 21 st Century. International Journal of Environmental Research and Public Health, 13(11), 1065. DOI: https://doi.org/10.3390/ijerph13111065

Moraru, R. (2012). Current trends and future developments in occupational health and safety risk management. Risk Management for the Future, Theory and Cases. London: Jan Emblemsvåg, IntechOpen. DOI: https://doi.org/10.5772/31577

Moreno, F. \& Godoy, E. (2012). Riesgos laborales un nuevo desafío para la gerencia. International Journal of Good Conscience, 7(1), 38-56. Recuperado de www.spentamexico.org/v7-n1/7(1)3856.pdf

Nicolaci, M. (2008). Condiciones y medio ambiente de trabajo. Hologramática, 5(8), 3-48. Recuperado de http://cienciared.com.ar/ra/usr/3/591/hologramatica08_v2pp3_48.pdf

OIT (2011). Sistema de Gestión de la Seguridad y Salud en el Trabajo: Una herramienta para la mejora continua: Organización Internacional del Trabajo. Recuperado de http://www.ilo.org/wcmsp5/groups/public/---ed_protect/---protrav/--safework/documents/publication/wcms_154127.pdf 
Seguridad y salud laboral: una revisión en el contexto actual, a propósito de la nueva ISO 45.001

Ove-Hansson, S. \& Schenk, L. (2016). Protection without Discrimination: Pregnancy and Occupational Health Regulations. European Journal of Risk Regulation, 7(2), 404-412. DOI: https://doi.org/10.1017/S1867299X00005808

Reinert, D. (2016). The future of OSH: a wealth of chances and risks. Industrial Health, 54(5), 387388. DOI: https://doi.org/10.2486/indhealth.54-387

Riaño-Casallas, M.; Hoyos, E. \& Valero, I. (2016). Evolución de un Sistema de Gestión de Seguridad y Salud en el Trabajo e Impacto en la Accidentalidad Laboral: Estudio de Caso en Empresas del Sector Petroquímico en Colombia. Ciencia \& Trabajo, 18(55), 68-72. Recuperado de https://scielo.conicyt.cl/pdf/cyt/v18n55/art11.pdf

Rodríguez, E. (2010). Protección de la seguridad y salud de los trabajadores. Una revisión desde la perspectiva global, latinoamericana y venezolana. Ingeniería Industrial. Actualidad y Nuevas Tendencias, 2(5), 81-96. Recuperado de http://www.redalyc.org/articulo.oa?id=215016943006 Rojas, L.; Sanabria, C.; Pedraza, E.; Quevedo, A.

\& Rojas, L. (2014). Propuesta de modelo de gerencia estratégica para un servicio de seguridad y salud en el trabajo tipo mancomunado. Formación Gerencial, 13(1), 58-87. Recuperado de http://produccioncientificaluz.org/index.php/rafg/article/view/6638/6627

Romeral, J. (2012). Gestión de la seguridad y salud laboral, y mejora de las condiciones de trabajo. El modelo español. Boletín Mexicano de Derecho Comparado, 45(135), 1325-1339. Recuperado de: http://www.redalyc.org/articulo.oa? id=42724584012

Sembe, F. \& Ayuo, A. (2017). Effect of Selected Occupational Health and Safety Management Practices on Job Satisfaction of Employees in University Campuses in Nakuru Town, Kenya. Journal of Human Resource Management, 5(5), 70-77. DOI: https://doi.org/10.11648/j.jhrm.20170505.11 Sparks, K.; Faragher, B. \& Cooper, C. (2001). Wellbeing and Occupational Health in the 21st Century Work Place. Journal of Occupational and Organizational Psychology, 74, 489-509. DOI:

https://doi.org/10.1348/096317901167497

Suárez, F.; Carvajal, G. \& Catalá, J. (2017). Occupational safety and health in construction: a review of applications and trends. Industrial health, 55(3), 210-218. DOI: https://doi.org/10.2486/indhealth.2016-0108

Zepeda, D.; Munguía, N. \& Velazquez, L. (2015). The importance of occupational safety and health in management systems in the construction industry: case study of construction in Hermosillo. Central and Eastern European Journal of Management and Economics, 3(1), 51-69. Recuperado de 
Seguridad y salud laboral: una revisión en el contexto actual, a propósito de la nueva ISO 45.001

http://cejsh.icm.edu.pl/cejsh/element/bwmeta1.element.desklight-f4804c4c-ebf9-4e35-8224ea38ad51f809

Zohreh, M. \& Napsiah, N. (2014). The relationship between occupational safety, health, and environment, and sustainable development: a review and critique. International Journal of Innovation, Management and Technology, 5(3), 198-2002. DOI: https://doi.org/10.7763/IJIMT.2014.V5.513. 